\title{
Protocol implementation in anesthesia: beta-blockade in non-cardiac surgery patients
}

\author{
[Application d'un protocole en anesthésie : les bêta-bloquants chez les patients en \\ chirurgie non cardiaque]
}

Alan D. Baxter MD FRCPC, ${ }^{*}$ Salmaan Kanji PharmD $†$

Purpose: An audit of intensive care unit (ICU) patients with perioperative myocardial ischemia and/or infarction (PMI/I) suggested under-use of prophylactic beta-adrenergic blocking drugs (ABDs). A multidisciplinary team developed an institutional protocol to identify at-risk patients, to standardize and facilitate prophylactic beta-adrenergic blockade, and to improve management of such patients. We report a retrospective assessment of the efficiency of program implementation.

Methods: Eligible preanesthesia assessment unit patients received metoprolol for one to four weeks prior to surgery, intraoperatively, and postoperatively. Patients with PMI/I requiring ICU admission were tracked from January 2002 to December 2004. The protocol was implemented in May 2003. The efficiency of program implementation was evaluated during two months of normal operating room activity (September 2003 and February 2004).

Results: The use of ABDs increased during the audit. Preoperative use increased from 31\% in September 2003 to $39 \%$ of eligible patients in February 2004, with a stable surgical population. The incidence of patients with $\mathrm{PMI} / \mathrm{I}$ admitted to ICU decreased from 2.6/I,000 surgical cases pre-implementation to $1.6 / 1,000$ surgical cases post-implementation $(P=$ $0.025)$. For the whole hospital, implementation was associated with a decrease in $\mathrm{PMI} / \mathrm{I}$ incidence from 5.9 to $2.0 / \mathrm{I}, 000$ surgical cases $(P<0.001)$.

Conclusion: Heightened awareness and standardization of perioperative beta-blockade coincided with an increase in metoprolol use in at-risk patients and reduction in PMI/I. There was an increase in at-risk patients receiving prophylactic ABDs, a reduction in $\mathrm{PMI} / \mathrm{l}$ diagnoses throughout the hospital, and reduced ICU patient admissions with $\mathrm{PMI} / \mathrm{I}$.

CAN J ANESTH $2007 / 54: 2 /$ pp 114-123
Objectif: Un audit de patients d'unité des soins intensifs (USI) souffrant d'ischémie myocardique et / ou d'infarctus (IMP/I) périopératoire suggère que les bêta-bloquants sont sous-utilisés en prophylaxie. Une équipe pluridisciplinaire a développé un protocole institutionnel afin d'identifier les patients à risque, de standardiser et faciliter la prophylaxie avec bêta-bloquants, et d'améliorer la prise en charge de tels patients. Nous présentons une évaluation rétrospective de l'efficacité de l'implantation du programme.

Méthodes : Les patients éligibles vus à la clinique préanesthétique ont reçu du métoprolol de une à quatre semaines avant la chirurgie, pendant la chirurgie et après la chirurgie. Les patients avec IMP/I nécessitant une admission aux soins intensifs ont été suivis de janvier 2002 à décembre 2004. Le protocole a été mis en place en mai 2003. L'efficacité de l'application du programme a été évaluée durant deux mois d'activité normale du bloc opératoire (septembre 2003 et février 2004).

Résultats : L'utilisation de bêta-bloquants a augmenté pendant l'audit. L'utilisation préopératoire est passée de $31 \%$ en septembre 2003 à 39\% des patients éligibles en février 2004, sur une population chirurgicale stable. L'incidence de patients avec IMP/I admis en USI a diminué de 2,6/I 000 cas chirurgicaux avant la mise en place du protocole à I,6/I 000 cas après sa mise en place $(P=0,025)$. Dans tout l'hôpital, la mise en place a été associée à une diminution de cas de IMP/I de 5,9 à 2,0/I 000 cas chirurgicaux $(P<0,001)$.

Conclusion: Une attention plus grande et une standardisation des bêta-bloquants en période périopératoire ont coïncidé avec une augmentation de l'utilisation du métoprolol chez les patients à risque et une réduction des IMP/I. II y a eu une augmentation de patients à risque recevant des bêta-bloquants en prophylaxie, une réduction des diagnostics de IMP/I dans tout l'hôpital, et une diminution des admissions à l'USI pour IMP/I.

From the Department of Anesthesia and Critical Care, ${ }^{*}$ The Ottawa Hospital; and the Departments of Pharmacy and Critical Care, $†$ The Ottawa Hospital and Ottawa Health Research Institute, Ottawa, Ontario, Canada.

Address correspondence to: Dr. Alan Baxter, Department of Anesthesia, The Ottawa Hospital, General Campus, 501 Smyth Rd., Ottawa, Ontario K1H 8L6, Canada. Phone: 613-737-8187; Fax: 613-737-8189; E-mail: abaxter@ottawahospital.on.ca

Declaration of funding: None.

Declaration of conflict of interest: None

Presented in part as poster presentations at the CAS Annual Meeting and Toronto Critical Care Symposium, both in 2004

Accepted for publication April 4, 2006.

Revision accepted August 7, 2006.

Final revision accepted November 16, 2006.

CAN J ANESTH 54: 2 www.cja-jca.org February, 2007 
$\mathrm{T}$ HERE is often a long delay between the acquisition of knowledge from research and its introduction into routine clinical practice. ${ }^{1}$ However, the implementation of often simple and inexpensive measures can lead to gratifying improvements in patient outcomes. ${ }^{2}$

While clinical trials describe technological and pharmacological interventions that provide mortality and morbidity benefits, there are many barriers to clinical implementation, including lack of awareness, cost, and potential risk. Assessment and understanding of risks and potential benefits of new therapies are crucial and require familiarity with the clinical trials supporting their use, for appropriate patient selection and accurate intervention. A strategy to involve protocolization is frequently successful in implementing new evidence-based therapy in a standardized and safe manner. ${ }^{3-5}$ Evidence-based protocols developed by multidisciplinary teams complement clinical judgment, standardize interventions and enhance routine clinical care. However, successful implementation of protocols requires stakeholder acceptance, education, training, accessible support systems, and ongoing evaluation. We describe here the implementation and protocolization of beta-adrenergic blockade drugs (ABDs) for at-risk surgical patients receiving care at a single tertiary care adult hospital.

\section{Methods}

\section{Identification of the problem}

The Ottawa Hospital, General Campus, is one of the two major in-hospital sites of an adult tertiary care teaching hospital. Approximately 900-1,000 patients undergo surgical procedures each month, involving general, thoracic, otolaryngologic, gynecologic, oncologic, ophthalmic, plastic and orthopedic surgeries; $60 \%$ are same-day-admission patients and $21 \%$ are non-elective. Postoperatively, a diagnosis of perioperative myocardial infarction or cardiac ischemia (PMI/I) was made either in the postanesthesia care unit or the intensive care unit (ICU). The diagnosis of myocardial ischemia was based on new ST segment depression $>1 \mathrm{~mm}$ at $\mathrm{J}+60 \mathrm{msec}$ without troponin elevation, whereas the diagnosis of myocardial infarction was made in the presence of ST depression/elevation/Twave inversion/new $\mathrm{Q}$ waves, in association with troponin elevation $>0.1 \cdot \mu \mathrm{g} \cdot \mathrm{L}^{-1}$. A prospective audit of a four-month (June to September 2002) period was performed and included all ICU patients who had surgery before admission to the ICU or during their stay in the ICU. The audit confirmed an increase in the frequency of ICU admissions with PMI/I, compared with the previous 18 months. The patients' charts were reviewed to verify the diagnosis, and to extract relevant clinical information. Seventeen patients were identified, 16 with myocardial infarction and one with ischemia. Sixteen events occurred between the intraoperative period and postoperative day five. The audit suggested an increase from an incidence of $\mathrm{PMI} / \mathrm{I}$ of 2.68 patients/1,000 surgical cases over the previous 18 months, to $4.25 / 1,000$ surgeries between June and September 2002 with a mortality rate of $42 \%$. Recent reports have described the prevention of $\mathrm{PMI} / \mathrm{I}$ using $\mathrm{ABDs}^{6-10}$ and other therapeutic modalities. ${ }^{1-14}$ Although $76 \%$ of PMI/I patients had documented risk factors, only $29 \%$ had received ABDs.

\section{Creating awareness of the problem}

We hypothesized that this higher incidence may have been the result of under-utilization of potentially useful prophylactic strategies. Perioperative ABDs were infrequently used in audit patients, and while their use for prophylaxis in surgical patients is currently controversial and more evidence is desirable, we felt it appropriate to attempt to increase their usage as a prophylactic strategy in at-risk patients. Representatives from anesthesia, critical care, internal medicine, nursing, pharmacy, family practice, and surgery formed a multidisciplinary team to develop a protocol to improve the preoperative identification of patients at risk and a tool to facilitate perioperative prophylaxis with ABDs. Increased educational efforts by the authors and other critical care physicians targeted anesthesiologists, critical care practitioners and trainees, surgeons, nurses and pharmacists. These consisted of formal presentations, informal teaching rounds, bedside discussions and one-on-one interactions to improve awareness of the problem and solicit opinions and possible solutions. Our objectives were to:

1) develop an institutional protocol to identify surgical patients at risk for PMI/I;

2) develop a tool to standardize and facilitate the administration of $\mathrm{ABDs}$ to at-risk surgical patients;

3) evaluate the acceptance and utilization of the program and continue to follow PMI/I rates after protocol implementation.

Our goal was not to evaluate the efficacy of ABDs in the setting of a clinical trial. We critically evaluated the published literature on perioperative ABDs, and determined that it supported their prophylactic use in a specific "at-risk" population, with a standardized protocol, appropriate patient selection, periodic evaluation of efficiency and safety, and modification when 
necessary with information when available from new clinical trials. Such a clinical trial would require prospective randomization and rigorous patient monitoring to record all episodes of ischemia/infarction. We documented only those clinically apparent at the time to the patient caregivers.

\section{Identification of surgical patients at-risk for PMI/I}

At-risk patients were identified using Lee's revised cardiac risk index ${ }^{15}$ modified to include age (age $>70$ yr being common among audit patients with $\mathrm{PMI} / \mathrm{I}$ ), and major arthroplasty ${ }^{8,16}$ (Appendix 1). Because 90\% of ICU patients with PMI/I during the three-year period 2002-2004 had $\geq 2$ risk factors, these patients were considered eligible for the protocol. Exclusion criteria included: documented metoprolol allergy or severe adverse reaction, severe asthma or chronic obstructive pulmonary disease, congestive heart failure (New York Heart Association functional class IV), symptomatic conduction abnormalities, uncontrolled diabetes mellitus, symptomatic peripheral vascular disease, bradycardia (heart rate $<60 \cdot \mathrm{min}^{-1}$ ), second or third degree heart block, bifascicular block, and advanced liver failure.

\section{Protocol development}

The protocol was developed through collaboration between the members of the multidisciplinary group in order to facilitate buy-in from stakeholders and address issues and concerns from all disciplines. Eligible patients identified by the anesthesiologist in the preanesthesia assessment unit (PAU) were prescribed metoprolol using a pre-printed form (Appendix 2), which included the eligibility criteria, and received metoprolol $25-50 \mathrm{mg}$ orally twice daily for up to four weeks before surgery. The lower dose of $25 \mathrm{mg}$ twice daily orally was used in patients $>80 \mathrm{yr}$ of age, with a heart rate $<70 \cdot \mathrm{min}^{-1}$, or already on antihypertensive medications or calcium channel blocking drugs. Patients already taking ABDs continued their usual medication. High-risk patients (i.e., severe heart failure, etc.) were referred for cardiology assessment. The PAU physician dispensed metoprolol tablets from the hospital pharmacy at no cost to the patient. An information sheet was given to the patient and faxed to the family doctor.

Eligible patients not already taking $\mathrm{ABDs}$ were treated in the operating room or postanesthesia care unit (PACU) with iv metoprolol. Anesthesia management was at the discretion of the anesthesiologist, and included iv metoprolol, targeting a heart rate of $60-70 \cdot \mathrm{min}^{-1}$ pre-induction and during surgery. Postoperatively in the PACU and ICU, heart rate was controlled using iv metoprolol or esmolol by bolus or infusion with the same target, plus oral, nasogastric, or rectal metoprolol. High-risk patients were monitored in the PACU or transferred to the ICU as appropriate. On the nursing units, enteral metoprolol dosage was adjusted by nurses using a titration algorithm (Appendix 2), including extra doses as required for a target heart rate of $60-70 \cdot \mathrm{min}^{-1}$. Follow-up, dosage adjustment, documentation, and management of clinical problems if necessary were handled by the acute pain service (APS) anesthesiologist using the copy of the pre-printed order sheet to identify patients for follow-up, and to record changes and problems.

At hospital discharge, a prescription for metoprolol for one month was issued with other discharge medications by the surgeon. Further treatment was subsequently decided upon by the patient's family physician.

\section{Protocol implementation}

Since the protocol was complex and required collaboration between the patient, nurses, physicians, anesthesiologists and pharmacists, a number of strategies were used before and during implementation. Education included formal presentations at anesthesia and surgical rounds describing goals and implementation, and teaching sessions each month during ICU resident rotations. Prescribers were surveyed to ensure that the protocol was accepted. One-on-one discussions were initiated when appropriate to solicit feedback. The PAU nurses and physicians were encouraged to flag the charts of at-risk patients who met the protocol criteria. Program audits were presented as posters at anesthesia and critical care meetings, and as displays in the PAU, anesthesia department, PACU, and pharmacy.

\section{Evaluation of protocol introduction}

The primary goal of the evaluation of the protocol was to assess protocol utilization. Secondarily, the incidence of PMI/I was also evaluated as a measure of protocol efficiency.

The protocol was implemented in May 2003. Operating room records were audited twice after protocol implementation, in September 2003 and February 2004, during months of full operating room activity without holiday closures. Charts of patients were reviewed to determine the surgical procedures, the prevalence of risk factors, and perioperative ABDs usage. Follow-up sheets returned to the anesthesia department were reviewed to determine protocol usage and problems. Overall rates of metoprolol prescribing within the hospital were obtained from the hospital pharmacy database. 


\section{Trends in Hospital-wide Metoprolol Prescribing}

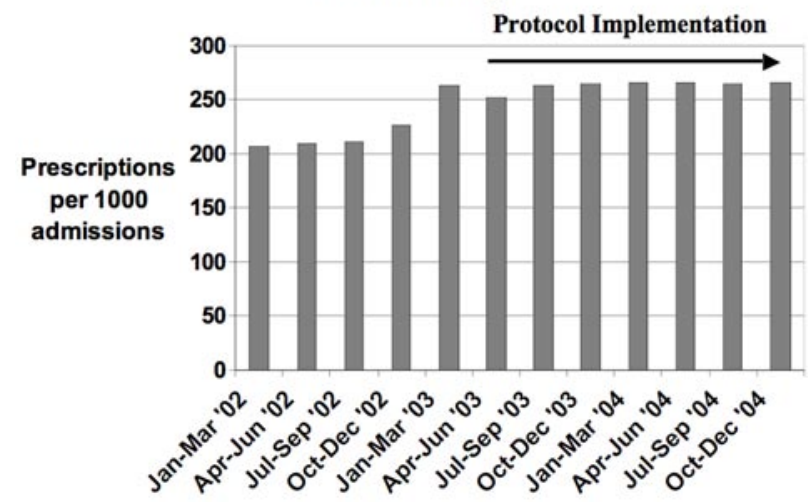

FIGURE 1 Trends in hospital-wide metoprolol prescribing Metoprolol prescriptions increased from $458 \pm 42$ before to $549 \pm 41$ per month after protocol implementation $(P$ $<0.001)$. A pre-implementation audit was performed from June to September 2002; post-implementation audits were performed in September 2003 and February 2004.

\section{Analysis}

The ICU PMI/I patients were identified using the ICU and hospital data bases, for January 2002 to April 2003, prior to protocol implementation, and May 2003 to December 2004 after protocol implementation. The PMI/I rates before and after protocol implementation were recorded as cases/1,000 surgeries $[95 \%$ confidence interval (CI)] and compared using the Chi-squared statistic. Patient charts were reviewed with Research Ethics Board approval to confirm the diagnosis, identify risk factors and determine eligibility for or receipt of beta-blocker therapy according the protocol. Incidence of PMI/I within the whole hospital, in surgical patients only, was obtained from hospital health records. This includes all patients whose physicians diagnosed PMI/I, without chart review verification.

\section{Results}

At least 334 patients were enrolled in the protocol, but only 128 follow-up sheets were returned to the anesthesia office for follow-up by the APS; 56 were from PAU and 72 were from the operating room. Clearly there were problems with this aspect of the protocol logistics.

The number of patients receiving ABDs hospitalwide increased during study period (Figure 1). In the two audited months after protocol implementation the
TABLE Incidence of risk factors in the two audited months

\begin{tabular}{lll}
\hline Factor & $\begin{array}{l}\text { September 2003 } \\
\text { (\% of patients) }\end{array}$ & $\begin{array}{l}\text { February 2004 } \\
\text { (\% of patients) }\end{array}$ \\
\hline Age > 70 yr & $27 \%$ & $29 \%$ \\
High/intermediate risk surgery & $45 \%$ & $46 \%$ \\
Coronary artery disease & $8 \%$ & $10 \%$ \\
Congestive cardiac failure & $2 \%$ & $7 \%$ \\
Diabetes mellitus & $9 \%$ & $10 \%$ \\
Chronic renal failure & $4 \%$ & $5 \%$ \\
Cerebrovascular disease & $5 \%$ & $5 \%$ \\
$\geq 2$ risk factors & $27.6 \%$ & $24.9 \%$ \\
\hline
\end{tabular}

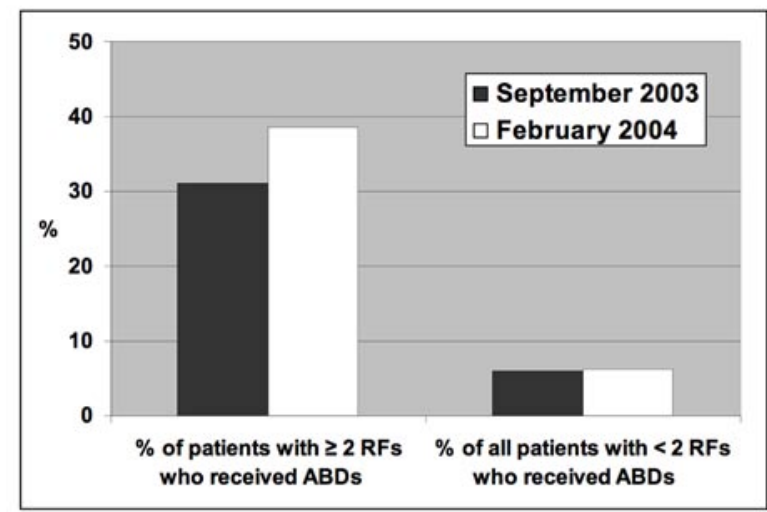

FIGURE 2 Risk factors and beta-adrenergic blocking drug $(\mathrm{ABD})$ use during two months of normal operating room activity. $\mathrm{RF}=$ risk factors.

number, types of surgeries, and patient risk factor distribution were similar, suggesting a stable surgical population (Table). However, the perioperative use of ABDs in eligible patients increased from $31 \%$ (September 2003 ) to $39 \%$ (February 2004) (Figure 2).

The hospital PMI/I incidence was reduced from 5.9 (95\% CI: $4.7-7.2)$ before, to 2.0 (95\% CI: 1.5-2.6) per 1,000 surgical cases $(P<0.001)$ after, protocol implementation. In ICU patients, the PMI/I incidence decreased from 2.6 (95\% CI: $1.8-2.4)$ to 1.6 (95\% CI: 1.1-2.1) per 1,000 surgical cases $(P$ $=0.025)$ after protocol implementation (Figure 3). All at-risk patients did not receive ABDs perioperatively despite the use of the protocol and the overall increased use in the hospital, but nevertheless the incidence of PMI/I was reduced. 


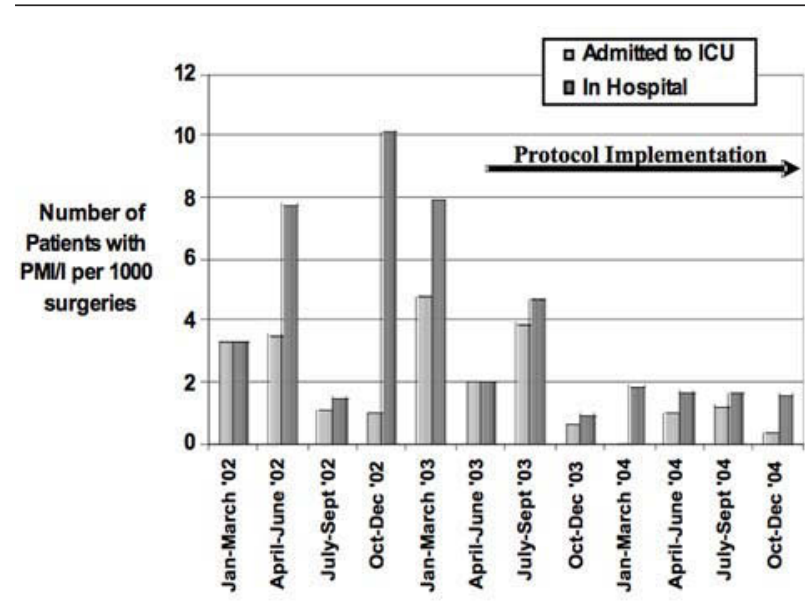

FIGURE 3 Prevalence of perioperative myocardial infarction/ischemia (PMI/I)

Pre-implementation audit performed from June to September 2002; post-implementation audits performed in September 2003 and February 2004. ICU = intensive care unit.

One patient enrolled in the protocol was admitted to the ICU with PMI/I. He received only one dose of metoprolol preoperatively, and no more until his myocardial infarction on day three. While absorption of metoprolol is primarily from the small intestine, ${ }^{17}$ the relatively few additional doses of metoprolol required using the titration schedule (eight recorded in 128 follow-up sheets) suggests that there was adequate gastric emptying in these patients for drug absorption to occur.

Side effects recorded on follow-up sheets included seven patients ( $5 \%$ of patients followed by APS) who had metoprolol doses held postoperatively: three for bradycardia, two for hypotension, one because "the patient's legs felt weak", and one whose family doctor felt the patient "reacted badly in the past". One patient with renal failure, metabolic acidosis and taking a non-steroidal anti-inflammatory analgesic became hyperkalemic. Dose adjustment during postoperative follow-up was required in only two patients.

\section{Discussion}

Delay between the discovery of useful therapies from research and their introduction into clinical practice has not been well studied in the perioperative period. Anesthesiologists usually practice independently and have relative freedom to implement evidence-based interventions in the operating room, within the limits of drug and equipment availability. However, the introduction of practices extending beyond the operating room involves complex interactions with other health care providers, and is facilitated by the use of protocols, education, etc. The anesthesiologist's involvement in preoperative assessment provides an opportunity for continued provision of perioperative care for higher acuity patients, many of whom receive follow-up care from anesthesiology for acute postoperative pain management.

We have confirmed the relatively low perioperative use of $\mathrm{ABDs}$ in at-risk patients recently documented $(38-40 \%)$ in another Canadian tertiary care institution. ${ }^{18}$ The accompanying editorial questioned the quality and quantity of evidence of efficacy of ABDs in preventing $\mathrm{PMI} / \mathrm{I},{ }^{19}$ supporting the need for a larger study. The numbers of patients in previous studies are relatively small (about 700 total), and there are unresolved questions regarding dosing and duration of therapy. However, current evidence suggests a therapeutic benefit and is intuitively reasonable, considering the body of evidence for ABDs in the cardiology literature. A recent meta-analysis ${ }^{20}$ concluded that "perioperative $\beta$ blockers may decrease the risk of major perioperative cardiovascular events" despite an increase in "the risk of bradycardia and hypotension needing treatment", "based on only a moderate number of major cardiovascular events", and hence justifying the performance of the PeriOperative ISchemic Evaluation (POISE) study. The authors of the meta-analysis do, however, concede that the evidence is "encouraging". The proposed intervention is inexpensive and carries a low risk, while the complication to be prevented is major, with significant health care costs and a high mortality. ${ }^{21} \mathrm{We}$ therefore decided to attempt to increase the perioperative use of $\mathrm{ABD}$ s by patients identified as being at increased risk of cardiac complications. Furthermore, protocolization of the intervention ensures standardization to minimize practice pattern variation and associated risks. The United States Agency for Healthcare Research and Quality (AHRQ) recommends"2 "The use of beta-blockers to reduce perioperative cardiac events and mortality represents a major advance in perioperative medicine for some patients at intermediate and high risk for cardiac events during non-cardiac surgery. Wider use of this therapy should be promoted and studied, with future research focused on fine-tuning dosages and schedules and identifying populations of patients in which its use is cost-effective". We decided for the above reasons to implement a beta-blocker program in a standardized fashion for at-risk patients as a potential solution for the high rate of PMI/I that we observed, rather than as an evaluation of beta-blockade efficacy in this population. 
The increasing involvement of anesthesiologists in PAUs provides the opportunity to identify and prescribe optimal prophylactic therapy to at-risk patients. Armanious $\mathrm{et} \mathrm{al.} .^{23}$ assessed patients for risk of perioperative cardiac complications and referred high-risk patients for medical or cardiology management. Of 100 patients referred, 69 were started on ABDs for one to 45 days preoperatively; 32 received $\mathrm{ABDs}$ in the PACU, and three patients suffered a perioperative myocardial infarction. The impact of this approach on the overall rate of perioperative cardiac problems was not reported. Extending this approach to intermediate risk patients would place a large burden on medical or cardiac physicians, and we felt that anesthesiologists could manage prophylaxis of these patients. In addition, it is not clear from existing literature what is the optimum time to start $\mathrm{ABDs}$ to obtain maximal benefit, a question yet to be resolved.

Barriers to the introduction of new treatments or guideline implementation have recently been described. ${ }^{24}$

1) Lack of agreement about the evidence may be encouraged by unbalanced critique of studies, without considering aspects such as the riskbenefit ratio and costs of the intervention. Lack of knowledge resulting from inadequate continuing medical education is an obvious barrier, and we addressed this by departmental presentations. Amongst Canadian anesthesiologists, 93\% were aware of the potential benefits of perioperative ABDs, but only $57 \%$ regularly use these drugs for patients with coronary artery disease, and only $34 \%$ continue them into the postoperative period. ${ }^{25}$

2) Lack of self-efficacy, which is one's ability to introduce the intervention, is especially relevant in the perioperative period, with many caregivers involved in varying locations and the potential for "turf wars".

3) Lack of outcome expectancy may be a problem, as anesthesiologists are accustomed to seeing immediate results of interventions, rather than a more subacute and indirect process, and most do not participate in ongoing patient care.

4) The inertia of previous practices may be difficult to overcome.

5) There may be external barriers such as lack of reminders during busy preoperative assessment clinics and workload issues.

6) Guideline-related barriers related to ease of use, lack of convenient tools and increased paper work.
7) Patient-related barriers requiring appropriate counselling about the objectives, and measures to improve compliance with medications..

8) Environmental barriers which may include problems with cooperation of other care givers, the supplies of paperwork, and medications.

Various strategies have been described to address these barriers. Cook et al. ${ }^{26}$ recommend first doing "an environmental scan" by audit or utilization review to document the starting place, present practice, and evaluate the magnitude of the problem. It is necessary to understand current behaviour before targeting the behaviour to be changed (why, what, when, where, and who). Effective change strategies are adopted based on the above, providing education, tools, feedback, and including all stakeholders in the process.

A similar approach was described by van Bokhoven et al. ${ }^{27}$ Problem analysis at the outset includes description of the problem, identification of barriers to and facilitators of change, and description of the target population. The design process includes specification of performance and intervention objectives, selection of methods and strategies, program design, and pretesting materials to be used.

Landry and Sibbald ${ }^{28}$ reviewed the effectiveness of various strategies to change behaviour. They found evidence of effectiveness for "audit and feedback" (e.g., review of physician processes of care and their outcomes), "academic detailing" (individual physician education to promote desirable aspects of practice), "local opinion leaders" (respected physicians assuming a leadership role locally), and "reminder systems" (e.g., computerized prompts in patient records). "Printed materials" (passive dissemination of information through lectures and printed materials) were considered to be ineffective. A multifaceted strategy including active measures is more likely to be effective than interventions that rely solely on passive information transfer. ${ }^{29,30}$

The approach we used was a composite of those described above, and was effective in our environment. We performed the initial four-month audit in response to a clinical impression of an increased incidence of PMI/I in ICU patients. While this was a snapshot, continuous data were available from the ICU database documenting the prevalence of the problem. The reason for this increase was not obvious from the audit, but while most other modalities with demonstrated benefit were in use, the audit suggested an under-utilization of ABDs and suggested an approach involving a change in clinical practice that might improve patient care and outcomes. 
We first documented and quantified the extent of the local problem, and presented this at anesthesia and surgery educational rounds. A system was already in place to track PMI/I, and we refined this to evaluate future incidents. A multidisciplinary team was assembled to develop a tool to facilitate beta-blockade of selected patients using a simple and locally relevant triage system. Physicians and nurses involved in perioperative patient care were sensitized to the problem and protocol implementation using presentations at rounds, memos, etc. Feedback was solicited to seek out implementation barriers, which were addressed by flagging charts, and further teaching. Several anesthesiologists initially felt uncomfortable prescribing outpatient medications, even though the patient information and contact numbers provided were consistent with routine outpatient clinic or primary care practice. Maintenance of improvements was achieved by providing feedback from ongoing data collection, reminders, rounds and meeting presentations. Protocol introduction included discussion of other modalities shown to reduce perioperative ischemia, e.g., epidural analgesia ${ }^{11}$ and maintenance of normothermia, ${ }^{12}$ and use of these strategies may have increased during the period of the audit. Hospital metoprolol utilization was tracked by the hospital pharmacy. Data were collected over a two-month period to reflect full operating room activities without holiday closures, before and after the documented change in incidence of $\mathrm{PMI} / \mathrm{I}$. These data were reviewed in detail to assess the surgical population, risk factors, and any change in ABD utilization. While there may have been variations from month to month, the data were consistent and favourable, suggesting that we were successful in targeting high-risk perioperative patients.

\section{Conclusions}

A multifaceted approach designed to change physician behaviour and increase the number of at-risk patients receiving $\mathrm{ABDs}$ when undergoing surgery is described. This involves passive elements such as education, and active elements including ongoing audits, provision of implementation tools, and ongoing reminders. We observed an increase in the proportion of at-risk patients receiving prophylactic $\mathrm{ABDs}$, and a reduction in the number of patients with PMI/I admitted to the ICU and throughout the hospital.

\section{References}

1 Levy MM, Pronovost PJ, Dellinger RP, et al. Sepsis change bundles: converting guidelines into meaningful change in behavior and clinical outcome. Crit Care Med 2004; 32(Suppl 11): S595-7.
2 Baxter AD, Allan J, Bedard J, et al. Adherence to simple and effective measures reduces the incidence of ventilator-associated pneumonia. Can J Anesth 2005; 52: 535-41.

3 Rivers E, Nguyen B, Havstad S, et al.; Early GoalDirected Therapy Collaborative Group. Early goaldirected therapy in the treatment of severe sepsis and septic shock. N Engl J Med 2001; 345: 1368-77.

4 Kanji S, Singh A, Tierney M, Meggison H, Mcintyre L, Hebert PC. Standardization of intravenous insulin therapy improves the efficiency and safety of blood glucose control in critically ill adults. Intensive Care Med 2004; 30: 804-10.

5 Kress JP, Pohlman AS, O'Connor MF, Hall JB. Daily interruption of sedative infusions in critically ill patients undergoing mechanical ventilation. N Engl J Med 2000; 342: 1471-7

6 Mangano DT, Layug EL, Wallace A, Tateo I. Effect of atenolol on mortality and cardiovascular morbidity after noncardiac surgery. Multicenter Study of Perioperative Ischemia Research Group. N Engl J Med 1996; 335: 1713-20.

7 Poldermans D, Boersma E, Bax JJ, et al. The effect of bisoprolol on perioperative mortality and myocardial infarction in high-risk patients undergoing vascular surgery. Dutch Echocardiographic Cardiac Risk Evaluation Applying Stress Echocardiography Study Group. N Engl J Med 1999; 341: 1789-94.

8 Urban MK, Markowitz SM, Gordon MA, Urqubart BL, Kligfield $P$. Postoperative prophylactic administration of beta-adrenergic blockers in patients at risk for myocardial ischemia. Anesth Analg 2000; 90: 1257-61.

9 Raby KE, Brull SJ, Timimi F, et al. The effect of heart rate control on myocardial ischemia among high-risk patients after vascular surgery. Anesth Analg 1999; 88: 477-82.

10 Stone JG, Foex P, Sear JW, Johnson LL, Khambatta HJ, Triner L. Myocardial ischemia in untreated hypertensive patients: effect of a single small oral dose of a betaadrenergic blocking agent. Anesthesiology 1988; 68: 495-500.

11 Rodgers A, Walker N, Schug S, et al. Reduction of postoperative mortality and morbidity with epidural or spinal anaesthesia: results from overview of randomised trials. BMJ 2000; 321: 1493-1504.

12 Frank SM, Fleisher LA, Breslow MJ, et al. Perioperative maintenance of normothermia reduces the incidence of morbid cardiac events. JAMA 1997; 277: 1127-34.

13 Stuhmeier KD, Mainzer B, Cierpka J, Sandmann W, Tarnow J. Small, oral dose of clonidine reduces the incidence of intraoperative myocardial ischemia in patients having vascular surgery. Anesthesiology 1996; 85: 706-12. 
14 McSPI-Europe Research Group. Perioperative sympatholysis: beneficial effects of the alpha-2 adrenoceptor agonist mivazerol on hemodynamic stability and myocardial ischemia. Anesthesiology 1997; 86: 346-63.

15 Lee TH, Marcantonio ER, Mangione CM, et al. Derivation and prospective validation of a simple index for prediction of cardiac risk of major noncardiac surgery. Circulation 1999; 100: 1043-9.

16 Eagle KA, Berger PB, Calkins H, et al. ACC/AHA guideline update for perioperative cardiovascular evaluation for noncardiac surgery - executive summary a report of the American College of Cardiology/ American Heart Association Task Force on Practice Guidelines (Committee to Update the 1996 Guidelines on Perioperative Cardiovascular Evaluation for Noncardiac Surgery). Circulation 2002; 105: 1257-67.

17 Jobin G, Cortot A, Godbillon J, et al. Investigation of drug absorption from the gastrointestinal tract of man. I. Metoprolol in the stomach, duodenum and jejunum. Br J Clin Pharmacol 1985; 19(Suppl 2): 97S-105S.

18 Rapchuk I, Rabuka S, Tonelli M. Perioperative use of beta-blockers remains low: experience of a single Canadian tertiary institution. Can J Anesth 2004 51: 761-7.

19 Devereaux PJ, Leslie $K$, Yang $H$. The effect of perioperative beta-blockers on patients undergoing noncardiac surgery - is the answer in? Can J Anesth 2004; 51: 749-55.

20 Devereaux PJ, Beattie WS, Choi PT, et al. How strong is the evidence for the use of perioperative beta blockers in non-cardiac surgery? Systemic review and metaanalysis of randomised controlled trials. BMJ 2005; 331: 313-21.

21 Auerbach AD, Goldman L. $\beta$-Blockers and reduction of cardiac events in noncardiac surgery: scientific review. JAMA 2002; 287: 1435-44.

22 Auerbach $A D$. Beta blockers and reduction of perioperative cardiac events. In: AHRQ Publication No. 01E058, Rockville, MD: Agency for Healthcare Research and Quality; July 2001. Available from URL; http:// www.ahrq.gov/clinic/ptsafety/chap25.htm.

23 Armanious S, Wong DT, Etchells E, Higgins P, Chung $F$. Successful implementation of perioperative betablockade utilizing a multidisciplinary approach. Can J Anesth 2003; 50: 131-6.

24 Cabana MD, Rand CS, Powe NR, et al. Why don't physicians follow clinical practice guidelines? A framework for improvement. JAMA 1999; 282: 1458-65.

25 VanDenKerkhof EG, Milne B, Parlow JL. Knowledge and practice regarding prophylactic perioperative beta blockade in patients undergoing noncardiac surgery: a survey of Canadian anesthesiologists. Anesth Analg 2003; 96: 1558-65.
26 Cook DJ, Montori VM, McMullin JP, Finfer SR, Rocker $G M$. Improving patients' safety locally: changing clinician behaviour. Lancet 2004; 363: 1224-30.

27 van Bokboven MA, Kok G, van der Weijden T. Designing a quality improvement intervention: a systematic approach. Qual Saf Health Care 2003; 12: 215-20.

28 Landry MD, Sibbald WJ. Changing physician behavior: a review of patient safety in critical care medicine. J Crit Care 2002; 17: 138-45.

29 Majumdar SR, Soumari SB. Why most interventions to improve physician prescribing do not seem to work. CMAJ 2003; 169: 30-1.

30 Grimshaw JM, Sirran L, Thomas R, et al. Changing provider behavior: an overview of systematic reviews of interventions. Med Care 2001; 39(8 Suppl 2): II2-45.

\section{APPENDIX 1}

Eligibility criteria for use of perioperative beta-adrenergic blockade

Two or more of:

- Age $\geq 70 \mathrm{yr}$

- High/intermediate risk surgical procedure, defined as $i p$, intrathoracic, major arthroplasty, or suprainguinal vascular procedure

- Ischemic heart disease: previous myocardial infarction/coronary artery surgery/angioplasty, past/present angina

- Heart failure, past or present

- Cerebrovascular disease: transient ischemic attack or cerebrovascular accident

- Diabetes mellitus

- Chronic renal insufficiency - baseline creatinine level $\geq 177 \mu \mathrm{mol} \cdot \mathrm{L}^{-1}\left(2.0 \mathrm{mg} \cdot \mathrm{dL}^{-1}\right)$ 
APPENDIX 2 Pre-printed order sheet

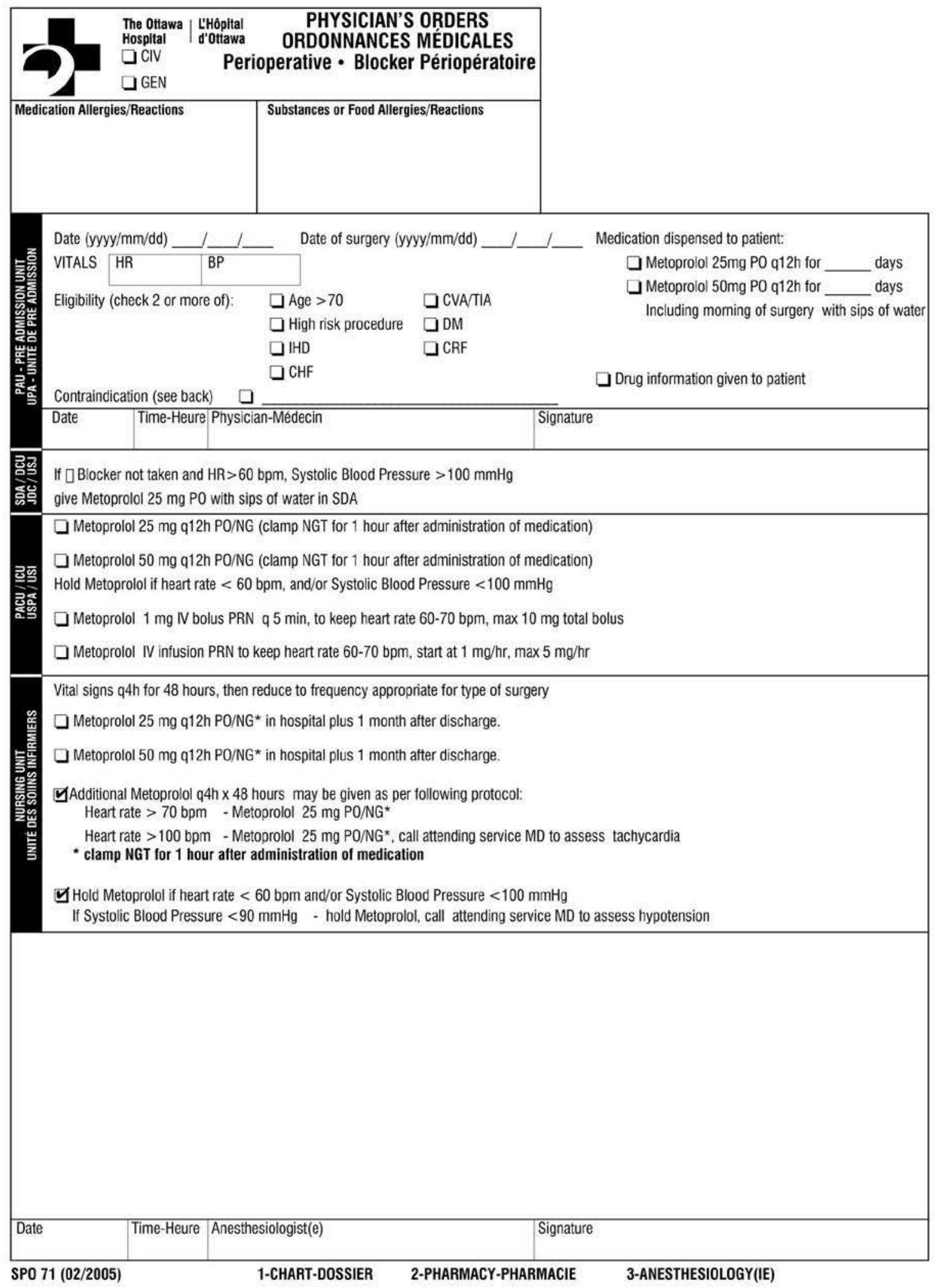




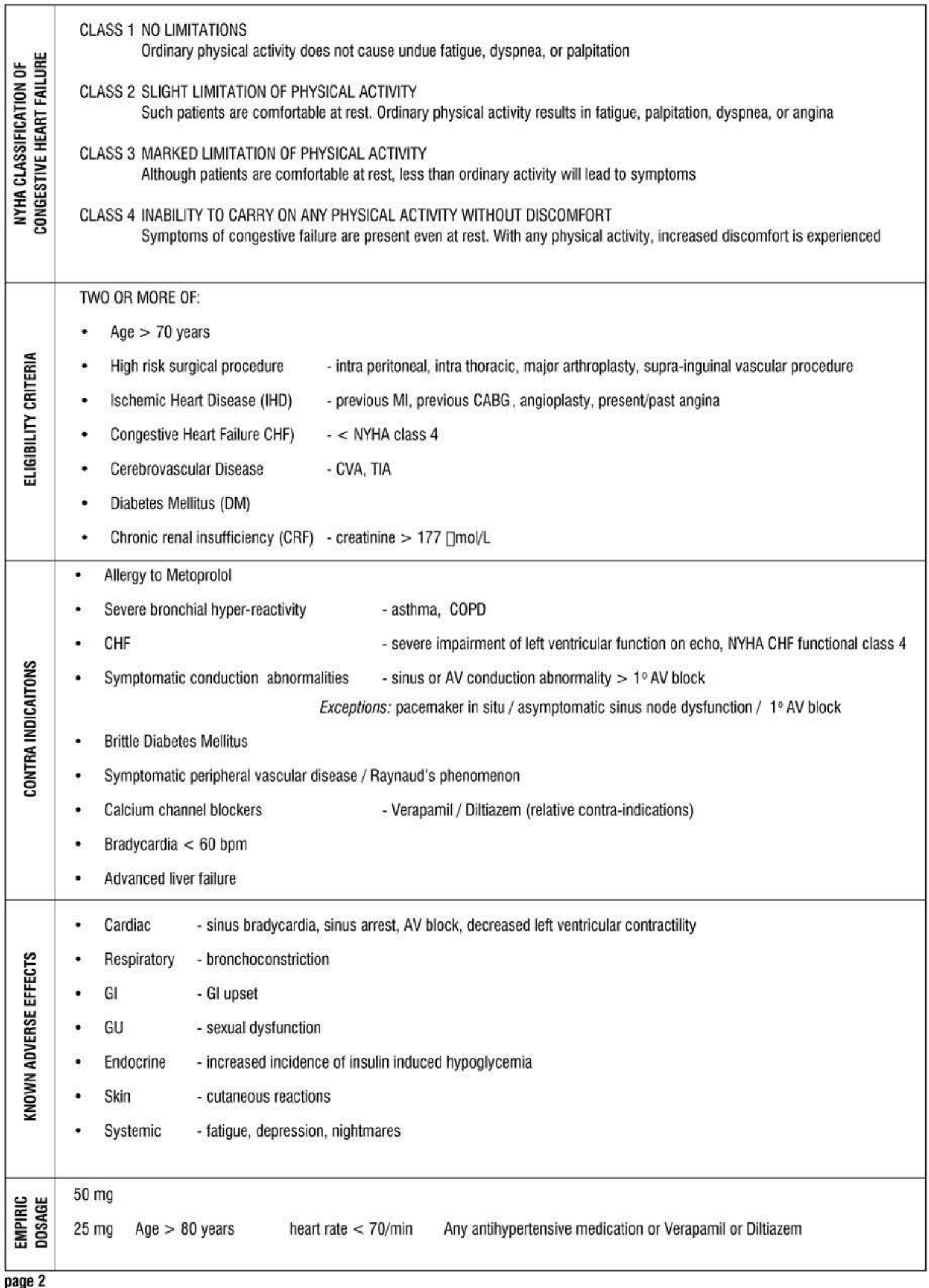

